\title{
Myasthenic crisis treated in a Chinese neurological intensive care unit: clinical features, mortality, outcomes, and predictors of survival
}

\author{
Fan Liu ${ }^{2+}$, Qiong Wang ${ }^{3+}$ and Xueping Chen ${ }^{1 *}$ (B)
}

\begin{abstract}
Background: Myasthenic crisis (MC) often requires admission to an intensive care unit (ICU).

Methods: We retrospectively investigated 113 consecutive patients with first MC admitted to the neurological ICU. Patients' demographic, clinical and other characteristics were examined, as well as therapeutic interventions, mortality and functional outcome.

Results: MC patients at first onset admitted to neurological ICU had a mortality rate of $18.6 \%$. $\mathrm{PCO}_{2}$ level before intubation and score on Myasthenia Gravis-Activities of Daily Living (MG-ADL) scale at MC onset correlated with duration of ventilation and length of ICU stay. Compared with patients with good functional outcome, patients with intermediate or poor functional outcome were older at first $\mathrm{MC}$ onset, had lower $\mathrm{pH}$ and $\mathrm{PO}_{2}$, and had higher $\mathrm{PCO}_{2}$ before intubation. Multivariate logistic analysis identified pre-intubation $\mathrm{PCO}_{2}$ level as an independent predictor of survival. Cox regression showed that age at first MC onset requiring ICU management was the factor which significantly influenced the mortality.

Conclusions: Our results suggest that $\mathrm{PCO}_{2}$ before intubation and $\mathrm{MG}-\mathrm{ADL}$ score at $\mathrm{MC}$ onset may be useful indicators of more severe disease likely to require extensive respiratory support and ICU management. Higher pre-intubation $\mathrm{PCO}_{2}$ indicates chronic respiratory acidosis that can increase risk of severe disability and death, especially in patients with older age at first $\mathrm{MC}$ onset.
\end{abstract}

Keywords: Myasthenic crisis, Intensive care unit, Clinical characteristics, Management, Outcomes, Survival predictor

\section{Background}

Myasthenia gravis (MG) is a neuromuscular disorder, characterized by muscle weakness and easy fatigability upon exertion. It is caused by the action of antibodies against proteins in the neuromuscular junction, and the most common autoantibody is the anti-acetylcholine receptor (AChR) antibody. This antibody reduces the number of postsynaptic acetylcholine receptors available on the end plate of the skeletal muscle. The second most prevalent antibody recognizes muscle-specific kinase

\footnotetext{
* Correspondence: chenxueping0606@sina.com

${ }^{\dagger}$ Fan Liu and Qiong Wang contributed equally to this work.

${ }^{1}$ Department of Neurology, West China Hospital, Sichuan University, 610041,

Guoxuexiang \#37, Chengdu, Sichuan, China

Full list of author information is available at the end of the article
}

(MuSK); this autoantibody is found in up to $70 \%$ of AChR-negative MG patients [1].

One of the most serious complications of MG is myasthenic crisis (MC), characterized by increased weakness of respiratory muscles that leads to acute respiratory failure requiring mechanical ventilation [2]. MC occurs in $10-20 \%$ of MG patients over the course of their disease [3-6]. While associated with mortality rates as high as $50-80 \%$ in the 1960 s, MC is now often reported to be fatal in fewer than $5 \%$ of cases as a result of the development of intensive care techniques $[2,6,7]$.

We undertook a retrospective study of MC patients with an exacerbation of MG admitted to the neurological intensive care unit (ICU) to understand more about the clinical characteristics, management and outcomes of

(c) The Author(s). 2019 Open Access This article is distributed under the terms of the Creative Commons Attribution 4.0 International License (http://creativecommons.org/licenses/by/4.0/), which permits unrestricted use, distribution, and 
patients with first $\mathrm{MC}$ who were requiring ICU management, We also performed regression analysis to identify baseline clinical and other factors that might predict survival for those MC patients.

\section{Methods}

\section{Patients}

The MG patients at our hospital were followed up since July 2008, including a cohort of 2023 MG patients. Patients had been diagnosed with MG because they showed two or more of the following clinical features: clinical evidence of muscle weakness and easy fatigability, significant decremental response on repetitive nerve stimulation, presence of anti-AChR or anti-MuSK antibodies, or objective clinical response to the neostigmine test [3]. Other diseases that mimic MG must be excluded, including Lambert-Eaton myasthenic syndrome, peripheral neuropathy, myopathies and motor neuron disease. MC was defined as respiratory failure from muscle weakness requiring mechanical ventilation with intubation or noninvasive ventilatory support (continuous or bi-level positive airway pressure) [3]. Patients were intubated if clinical assessment indicated compromised respiratory effort documented by decreased patient comfort with either single breath count less than 10, respiratory rate more than 30 per minute, decreased chest expansion, paradoxical diaphragmatic movements or arterial blood gas showing drop in oxygen saturation. Normal oxygen saturation does not exclude MC, and oxygen saturation drops late in neuromuscular respiratory failure. If life-threatening hypoxemia $\left(\mathrm{PaO}_{2}<60\right.$ $\mathrm{mmHg}$ ) occurs, and cannot be improved with supplemental oxygen, intubation is required. Furthermore, general criteria for intubation also included impaired swallowing mechanism leading to ineffective cough and nasal voice, inability to clear secretions. MG patients intubated for respiratory failure due to acute respiratory distress syndrome, congestive heart failure, and hypoxicischemic coma were excluded since in those cases respiratory muscle weakness was not a contributing factor for intubation. Therefore, we excluded one patient who was intubated for overwhelming asthma and one patient who was intubated following a cardiorespiratory arrest. Post-thymectomy crises were also excluded. In this retrospective study, patients admitted to the neurological ICU with first MC onset were investigated. MC patients managed only on the general ward were not included in the study. The decision to mechanically ventilate was taken on a case-by-case basis according to the patient's respiratory condition and with the consent of patients or relatives. Death of MC patients occurring at ICU and as well as at the residence were recorded. The period of evaluation of the patients was until May 2017.

\section{Data collection}

The following demographic data were recorded for each patient: gender, age of MG onset, age at first $\mathrm{MC}$ onset requiring ICU management, comorbidities, presence of antiAChR or -MuSK antibodies, and history of thymoma, thymectomy, and other autoimmune diseases. In addition, the following information was also collected: MG duration before MC, score on Myasthenia Gravis-Activities of Daily Living (MG-ADL) scale at disease onset, score on the Myasthenia Gravis Foundation of America (MGFA) scale at ICU admission, length of hospital stay, length of ICU stay, and duration of ventilator use. Primary precipitating factors were identified whenever possible; these included infection, surgery, medication, aspiration, pregnancy, and stressor. Possible secondary precipitating factors were also identified. Data was collected on the last time of arterial blood gas measurements before intubation, including white blood cell count (WBC), $\mathrm{Ph}, \mathrm{PO}_{2}$, and $\mathrm{PCO}_{2}$. Functional outcomes were assessed using the MGFA postintervention status every 3 months after discharge from the ICU. All included patients had follow-up duration of 12 months after discharged from ICU. In the present study, patients with different functional outcome were considered to experience good, intermediate, and poor prognosis. Good outcome was defined as achievement of complete stable remission (CSR), pharmacologic remission (PR) or minimal manifestations (MM). A status of improvement (IM) was categorized as intermediate outcome. Unchanged (U), worse (W), exacerbation (E) and died (D) were classified as poor outcome. CSR indicates no fatigable muscle weakness of MG for at least one year and being free of medication for MG. PR shows those MG patients taking some form of drug for MG excluding cholinesterase inhibitors, but with the same clinical criteria as for CSR. MM indicates MG patient who has no symptoms of functional limitations but has some weakness that is only detectable by careful examination. IM indicates a sustained substantial reduction in MG medications or substantial decrease in pretreatment clinical manifestations.

\section{Statistical analysis}

Descriptive statistics were used to evaluate clinical features. Data for continuous variables were compared between groups using the independent $t$ test, while data for categorical variables were compared using the chisquared or Fisher's exact tests. Correlation analyses were performed using Spearman's rank correlation test. The following variables were tested for their potential association with outcome: age of first $\mathrm{MC}$ onset requiring ICU management, arterial blood gas values, WBC, MG-ADL at $\mathrm{MC}$ onset, duration of ventilation and hospitalization, and ICU stay. Predictors of poor outcome were determined using logistic regression, followed by Cox regression to identify significant independent predictors. All 
analyses were performed using SPSS 18.0 (IBM, Chicago, IL) and a threshold of significance of $p<0.05$.

\section{Results}

\section{Demographic and clinical information}

During the study period, 113 patients (39 males, 74 females) suffered first MC onset requiring ICU management were admitted to our neurological ICU (Table 1). The rate of first MC was 5.6\%. Mean age at MG onset was 39.5 years (range, 15.2 to 73.6 years). Mean duration from MG onset to first MC onset requiring ICU management was 24.1 months (range, 1.2 to 110.5). Fiftyeight percent of patients experienced their first MC within one year after symptom onset. Mean age at first $\mathrm{MC}$ onset requiring ICU management was 40.5 years (range, 17.4 to 75.8 ), and 79 patients (20 males, 58 females) suffered first $\mathrm{MC}$ onset while younger than 50 (mean age at onset, 32.1). The remaining 36 patients (19 males, 17 females) experienced first MC onset when they were older than 50 (mean age at onset, 60.4).

All patients met at least one positive diagnostic test recognized in the inclusion criteria. The neostigmine test showed the highest overall sensitivity (88.0\%, 95/108 positive, 5 not tested), followed by the repetitive stimulation test (83.0\%, 83/100 positive, 13 not tested), and the anti-AChR autoantibody test $(67.4 \%, 62 / 92$ positive, 21 not tested) (Table 1).

\section{Precipitants of MC}

Infection was the most frequent precipitant of $\mathrm{MC}$, occurring in $51.3 \%$ of cases (58/113). This took the form of lower respiratory tract infection in 47 patients and upper respiratory infection in 11 patients. In addition, 8 of these 59 patients experienced both infection and hypokalemia. Precipitating factors due to medication occurred in 18 patients (15.9\%); 11 patients among them developed MC due to failure to comply with treatment, and $\mathrm{MC}$ in 7 patients was aggravated by high-dose steroid therapy. These 7 patients received oral steroid before crises, but acute deterioration following initiation of intra-venous methylprednisolone $1000 \mathrm{mg}$ /day treatment precipitated the ICU admission, and they showed symptoms of severe weakness of the respiratory and/or bulbar muscles and inability to maintain adequate ventilation. Three patients had MC during pregnancy (2.7\%), one patient developed $\mathrm{MC}$ with emotional upset after losing his family member $(0.9 \%)$, and one patient had MC after severe hypokalemia (0.9\%). No obvious precipitant was identified in the remaining 32 patients (28.3\%) (Table 1).

\section{Respiratory status, mechanical ventilation, and ICU stay} Included patients spent a mean of 12.2 days (range, 1 to 55 ) in the ICU and a mean of 26.3 days (range, 3 to 81) in the hospital overall. All patients received mechanical ventilation, for a mean duration of $190.3 \mathrm{~h}$ (range, 4 to 925). Respiratory status of all patients was evaluated prior to intubation, the last time of blood gas analysis before intubation indicated mean $\mathrm{pH}$ of 7.4 (range, 7.1 to 7.6), mean $\mathrm{PO}_{2}$ of $105.3 \mathrm{mmHg}$ (range, 56 to 220), and mean $\mathrm{PCO}_{2}$ of $48.4 \mathrm{mmHg}$ (range, 18.3 to 93.1). Leukocytosis was seen in 107 patients, and mean white blood cell count before intubation was $16.5 \times 10^{9} / \mathrm{L}$ (range, $8.0 \times 10^{9}$ to $25.8 \times 10^{9}$ ) (Table 2). At the time of MC onset, 38 (33.6\%) had mild symptoms (IIB), 62 patients (554.9\%) had moderate symptoms (MGFA IIIB), and $11(9.7 \%)$ had severe symptoms (IVB) (Table 2). In addition, 28 patients (24.8\%) received tracheostomies (MGFA V) at a mean of 10.5 days after presentation. The MGFA status of most patients progressively improved during follow-up. Mean MG-ADL score at first MC onset was 16.9 , and it correlated positively with $\mathrm{PCO}_{2}$ before intubation $(p=0.004)$. Several factors were assessed for possible association with duration of mechanical ventilation, hospital stay and ICU stay. Both $\mathrm{PCO}_{2}$ before incubation and MG-ADL score at first MC onset correlated positively with duration of ventilation and ICU stay. Longer duration of mechanical ventilation correlated positively with longer stay in the ICU and in the hospital (Table 3).

\section{Treatment}

Prior to admission to ICU, most patients received acetylcholinesterase inhibitors; and pyridostigmine bromide

Table 1 Baseline demographic and clinical data of patients with first MC onset

\begin{tabular}{ll}
\hline Parameter & value \\
\hline Age at MG onset (yr) & $39.5 \pm 16.7$ \\
Age at first MC onset (yr) & $40.5 \pm 16.1$ \\
Duration of MG before first MC (mo) & $24.1 \pm 25.3$ \\
Neostigmine Test (positive/negative/not tested) & $95 / 13 / 5$ \\
RNS (positive/negative/not tested) & $83 / 17 / 13$ \\
Anti-AChR auto-antibodies (positive/negative/not tested) & $62 / 30 / 21$ \\
Precipitant (Infection/Medication/Pregnancy/ Stressor/Hypokalemia without precipitant) & $58 / 18 / 3 / 1 / 1 / 32$
\end{tabular}

Abbreviations: MC myasthenic crisis, mo month(s), yr. year(s), RNS repetitive nerve stimulation 
Table 2 Laboratory tests, disease characteristics and outcomes of patients with first MC onset

\begin{tabular}{ll}
\hline Parameter & value \\
\hline Arterial blood gas before intubation & \\
$\mathrm{pH}$ & $7.4 \pm 0.1$ \\
$\mathrm{PO}_{2}(\mathrm{mmHg})$ & $105.3 \pm 43.6$ \\
$\mathrm{PCO}_{2}(\mathrm{mmHg})$ & $48.8 \pm 16.8$ \\
Blood test & \\
WBC (1099) & $16.5 \pm 6.2$ \\
Clinical severity & \\
MGFA (IIB/IIIB/IVB) & $38 / 62 / 11$ \\
MG-ADL scale score & $16.9 \pm 4.4$ \\
Duration on ventilation (hours) & $190.3 \pm 221.6$ \\
Duration of hospitalization (days) & $26.5 \pm 15.3$ \\
ICU stay (days) & $12.3 \pm 13.1$ \\
\hline Abbrevations ICU ins
\end{tabular}

Abbreviations: ICU intensive care unit, $M C$ myasthenic crisis, $M G-A D L$ Myasthenia Gravis-Activities of Daily Living scale, MGFA Myasthenia Gravis Foundation of America scale, mRS modified Rankin scale, WBC white blood cell count

was given to $101 \mathrm{MC}$ patients $(89.4 \%)$ before intubation. Four patients continued on a decreased dose after establishing mechanical ventilator support. Seventy-five patients were on oral prednisolone or intravenous methylprednisolone prior to admission to ICU (66.4\%). During stay in ICU, 104 patients were given steroids (92.0\%). Specifically, intra-venous methylprednisolone $1000 \mathrm{mg} /$ day was administered for 3-5 days, followed by oral prednisolone $(1 \mathrm{mg} / \mathrm{kg} /$ day $)$. During ICU staying, intravenous immunoglobulin (IVIG) therapy was given to 76 patients $(67.3 \%), 67$ of whom received steroid pulse IVIG combination therapy. Four patients received the combination of corticosteroids, IVIG, and plasma exchange. The remaining patients were unable to receive steroids or IVIG or plasma exchange because of hemodynamic instability or severe sepsis or the family's economic hardship. Other oral immunosuppressants such as tacrolimus were given to 12 patients (10.4\%).

Table 3 Factors associated with duration on ventilation, duration of hospitalization, and ICU stay

\begin{tabular}{lllllll}
\hline & \multicolumn{2}{l}{ duration on ventilation } & & \multicolumn{2}{l}{ ICU stay } & \\
\cline { 2 - 3 } \cline { 6 - 7 } & $r_{\mathrm{s}}$ & $P$ value & & $r_{\mathrm{s}}$ & $P$ value \\
\hline Age at first MC onset & 0.342 & 0.063 & & 0.232 & 0.168 \\
pH before intubation & -0.173 & 0.372 & & -0.145 & 0.487 \\
PO2 before intubation & -0.267 & 0.144 & & -0.213 & 0.239 \\
PCO2 before intubation & 0.319 & $\mathbf{0 . 0 4 3}$ & & 0.413 & $\mathbf{0 . 0 2 5}$ \\
MG-ADL at first MC onset & 0.541 & $\mathbf{0 . 0 0 2}$ & & 0.753 & $<\mathbf{0 . 0 0 0 1}$ \\
duration on ventilation & $/$ & $/$ & & 0.841 & $<\mathbf{0 . 0 0 0 1}$
\end{tabular}

Abbreviation: ICU intensive care unit; Significant values are highlighted in bold characters
Functional outcome, mortality, and comorbidities

For the prognosis, 87 patients $(77.0 \%)$ showed good outcome, 5 (4.4\%) had intermediate and 21 (18.6\%) had poor outcome. Eighteen patients died in the ICU; all these fatalities resulted from severe comorbidities that had kept them bed-ridden for a long time: pneumonia and respiratory failure $(n=7)$; bacteremia sepsis $(n=4)$; and uremia $(n=3)$ and heart failure $(n=3)$, followed by respiratory arrest $(n=1)$. Three patients died after discharge from the ICU: one patient's condition improved upon admission to the ICU, but she was not fully recovered, and she died 22 days later due to acute exacerbation of pneumonia after discharging from hospital. Two patients stopped treatment and requested early discharge because of financial problems. The twenty-one patients who died were significantly older than those who survived $(p=0.0009)$. Table 4 summarized the characteristics of all the included MC patients categorized to two outcome groups, those with good outcome $(n=87)$ and those with intermediate or poor outcome $(n=26)$. The mean age of first MC onset requiring ICU management was older in the intermediate or poor outcome group, the difference was statistically significant. Patients with intermediate or poor outcome had significantly lower $\mathrm{pH}$ and $\mathrm{PO}_{2}$ as well as significantly higher $\mathrm{PCO}_{2}$ in the last time of blood gas analysis before intubation, compared to patients with good outcome. In contrast; the two groups did not differ in WBC count or in the duration of ventilation, hospitalization, or ICU stay. Comparison of comorbidities between patients with good outcome and those with intermediate or poor outcome showed that there was no significant difference in comorbidities among patients with different outcomes (Table 4).

Univariate analysis using the log rank test identified the following four variables as significantly associated with survival: age at first $\mathrm{MC}$ onset requiring ICU management, gender, $\mathrm{PO}_{2}$, and $\mathrm{PCO}_{2}$ (Table 5). Older age of first $\mathrm{MC}$ requiring ICU management, male gender, lower $\mathrm{PO}_{2}$, and higher $\mathrm{PCO}_{2}$ were associated with higher mortality risk. However, multivariate logistic regression only identified pre-intubation $\mathrm{PCO}_{2}$ as an independent factor associated with survival. Higher pre-intubation $\mathrm{PCO}_{2}$ was associated with higher mortality risk. Cox regression identified that age at first MC onset requiring ICU management was the key factor which significantly influenced the mortality among the variables examined $(p=0.031)$.

\section{Discussion}

MC is a potentially life-threatening complication in patients with MG, but the mortality rate has fallen dramatically over the past 60 years. The introduction of the neurological ICU has substantially improved early recognition of $\mathrm{MC}$, identification of its precipitating factors 
Table 4 Comparison of patients showing good or poor outcome*

\begin{tabular}{|c|c|c|c|}
\hline & Good outcome $(n=87)$ & Intermediate or poor outcome $(n=26)$ & $P$ value \\
\hline Age at first $M C$ onset & $32.2 \pm 7.7$ & $54.2 \pm 15.9$ & $<0.0001$ \\
\hline $\mathrm{pH}$ & $7.4 \pm 0.6$ & $7.3 \pm 0.13$ & 0.0004 \\
\hline $\mathrm{PO} 2$ & $116.9 \pm 42.7$ & $80.2 \pm 32.5$ & 0.0396 \\
\hline PCO2 & $37.9 \pm 7.9$ & $58.6 \pm 20.8$ & 0.0005 \\
\hline WBC & $14.5 \pm 4.6$ & $16.1 \pm 5.4$ & 0.4557 \\
\hline Duration on ventilation, $\mathrm{h}$ & $168.0 \pm 233.9$ & $241.8 \pm 268.8$ & 0.2853 \\
\hline Duration of hospitalization, $\mathrm{d}$ & $21.3 \pm 15.2$ & $28.3 \pm 17.7$ & 0.5334 \\
\hline ICU stay, d & $10.2 \pm 16.3$ & $17.2 \pm 14.3$ & 0.1707 \\
\hline Comorbidities & $32.2 \pm 7.7$ & $54.2 \pm 16.0$ & $<0.0001$ \\
\hline Hypertension & 24 & 12 & 0.0565 \\
\hline $\mathrm{DM}$ & 10 & 6 & 0.1961 \\
\hline Hyperlipidemia & 9 & 4 & 0.4920 \\
\hline Heart disease & 3 & 1 & 1.0000 \\
\hline COPD & 3 & 2 & 0.3245 \\
\hline Stroke & 1 & 1 & 0.4088 \\
\hline Cancers & 4 & 1 & 1.0000 \\
\hline SLE & 3 & 0 & 0.1707 \\
\hline AID & 2 & 0 & 1.0000 \\
\hline Hepatitis & 5 & 2 & 0.6600 \\
\hline Hyperthyroidism & 2 & 0 & 1.0000 \\
\hline
\end{tabular}

Abbreviations: ICU intensive care unit, WBC white blood cell count, DM diabetic mellitus, SLE Systemic lupus erythematosus, AID autoimmune disease, COPD Chronic obstructive pulmonary disease; Significant values are highlighted in bold characters

and respiratory management of patients. The present work may help further improve the early recognition and care of patients who suffer MC by providing a picture of clinical characteristics (Additional file 1: Table S1) and even suggestions of baseline factors that may help predict survival.

Table 5 Predictors of death

\begin{tabular}{llll}
\hline & $\operatorname{Exp}($ Coef) & $95 \% \mathrm{Cl}$ & $P$ value \\
\hline Univariate & & & \\
Age at first MC onset & 1.135 & 1.040 to 1.238 & $\mathbf{0 . 0 0 4}$ \\
Gender & 8.500 & 1.247 to 57.931 & $\mathbf{0 . 0 2 9}$ \\
$\mathrm{PO}_{2}$ & 0.838 & 0.698 to 0.011 & 0.059 \\
$\mathrm{PCO}_{2}$ & 1.181 & 1.026 to 1.358 & $\mathbf{0 . 0 2 0}$ \\
WBC & 1.068 & 0.910 to 1.253 & 0.422 \\
MG-ADL at first MC onset & 1.176 & 0.949 to 1.459 & 0.139 \\
Duration of ventilation & 1.001 & 0.998 to 1.004 & 0.522 \\
Duration of hospitalization & 1.006 & 0.958 to 1.056 & 0.824 \\
ICU stay & 1.011 & 0.951 to 1.075 & 0.725
\end{tabular}

Multivariate

$$
\mathrm{PCO}_{2}
$$$$
1.147
$$$$
1.010 \text { to } 1.303
$$

Abbreviations: ICU intensive care unit, MG-ADL Myasthenia Gravis-Activities of Daily Living scale, WBC white blood cell count; Significant values are highlighted in bold characters
Mean age at first $\mathrm{MC}$ onset requiring ICU management was 40.5 years. However, the median age at first MC onset was 55 years in a US study [3]. One possible explanation for this discrepancy is ethnicity; other explanations include the differences in sample size, environmental factors and other population factors. In the present study, we found that first MC affecting people younger than 50 years affected women disproportionately, most of whom were aged 20-50; in contrast, first MC affecting people older than 50 did not show gender bias. These results are consistent with other studies $[3,6,8,9]$. The average interval from onset of $\mathrm{MG}$ to first $\mathrm{MC}$ requiring ICU management was 24.06 months in our cohort, much longer than the 8 months reported in another study [3]. Our results are consistent with recent reports of a median interval from onset to crisis of 3 years [9] and mean duration of $\mathrm{MG}$ prior to ICU admission of 3.8 years [10]. A longer interval from MG onset to first MC probably reflects recent improvements in recognition of the disease, management of respiratory and bulbar conditions, and greater access to newer treatment modalities. Just over half our patients experienced their first $\mathrm{MC}$ within one year of symptom onset, consistent with a study showing that MC typically occurs within the first 2 years after MG diagnosis [11].

While MG diagnosis in Europe and North America is most often supported using the tensilon test, the 
neostigmine test is used more often in China. In our study, the neostigmine test showed overall sensitivity of $88.0 \%$, a little bit lower compared to the $96.8 \%$ reported by another study in China [12]. These results validate the important role of this test for MG diagnosis in China. The proportion of patients in our cohort who took the repetitive stimulation test and gave a positive result was $83.00 \%$, higher than the $77.4 \%$ reported in a cohort of 1108 Chinese MG patients [12], and higher than the $75.9 \%$ reported in an Italian cohort [13]. The higher rate of positive results on the repetitive stimulation test in our study may reflect the fact that we included all MC patients admitted to the ICU during the study period, none of whom had ocular MG. Sixty-two patients in 92 cases were positive for anti-AChR antibodies. This may underestimate the real prevalence of such antibodies, since this test is not routine in China because of resource limitations.

Infection, especially lower respiratory tract infection, was the most common identifiable precipitant of $\mathrm{MC}$, followed by medication, and inadequate treatment/drug withdrawal. Other studies have also identified respiratory tract infection as the most frequent cause of $\mathrm{MC}$, accounting for about half of crises resulting in ICU admission $[9,10,14]$. Failure to comply with treatment or drug withdrawal was a frequent cause of $\mathrm{MC}$ accounting for 11 patients out of 113 in our study. Initial treatment with steroid led to exacerbation of MG in $30-50 \%$ of patients and decompensation in patients with $\mathrm{MC}$, whereas 9-18\% of them develop MC [15]. In the present study, 7 patients out of 113 develop MC due to high-dose steroid therapy. Therefore, initiation of high-dose steroid should occur in a hospital setting, where the respiratory function can be monitored [15]. Predictors of exacerbation from steroid include older age, bulbar symptoms, and lower score on Myasthenia Severity Scale [15-17]. Our study showed pregnancy as a trigger of $\mathrm{MC}$ being responsible for crisis in 3 out of 113 patients, and study reported that pregnancy can aggravate MG in 33\% of the MG cases [5, 18, 19]. We suggest a detailed review of systems when the disease is getting worse, with attention to infectious sources, respiratory symptoms, and drug exposures (12). Physicians must pay careful attention to respiratory rate, difficulty with phonation, a quiet voice, weak neck muscles, work of breathing, and oxygenation. If the patient demonstrates vital capacity $(\mathrm{VC})<10-20$ $\mathrm{mL} / \mathrm{kg}$ or negative inspiratory force $(\mathrm{NIF})<-20$ to -30 $\mathrm{cm} \mathrm{H} 2 \mathrm{O}$, diagnosis of $\mathrm{MC}$ is considered. However, these values are not derived from studies on patients with MG, but rather from studies in patients with GBS. We recommend that physicians should focus on the respiratory status of the patient, and trends in these symptoms, rather than relying on absolute numbers of VC or NIF. We also identified higher MG-ADL score at MC onset as a potential indicator that ICU care will be needed. Indeed, MG-ADL score $>18$ points at MC has been reported to predict the need for ICU management with $75 \%$ sensitivity and $77.8 \%$ specificity [14]. Surprisingly, we detected severe hypercarbia in our cohort before intubation (mean $\mathrm{PCO}_{2}, 48.78 \mathrm{mmHg}$ ). Since MG-ADL score at $\mathrm{MC}$ onset correlated positively with $\mathrm{PCO}_{2}$ before intubation, respiratory status may be tightly associated with MC symptoms, and hypercarbia may affect daily activities of patients with MC. Mean duration of ICU stay was 12.3 days in our study, similar to the median of 14 days reported in a US study thirty years ago [6] or the median of 13 days reported in a US study more recently [3]. The mean duration of ventilation of $190.3 \mathrm{~h}$ in our study is similar to the 8 days reported in a US study [20]. But a study from India showed that the median duration of ventilator was 14 days in a group of patients with MC [21], this result was similar to the previously reported duration of 13 days [22]. We found that pre-intubation $\mathrm{PCO}_{2}$ and MG-ADL score at MC onset were associated with duration of ventilation and ICU stay. Higher $\mathrm{PCO}_{2}$ prior to mechanical ventilation may indicate more severe condition that will likely require extensive respiratory support and ICU management. Respiratory management is important for $\mathrm{MC}$, one study showed that bilevel positive airway pressure (BiPAP) before the development of hypercapnia was useful in preventing intubation and prolonged ventilation [23]. Another study revealed that hypercapnia at onset predict BiPAP failure and subsequent intubation [24]. Therefore, high $\mathrm{PCO}_{2}$ level before intubation may be an independent predictor of prolonged intubation. Thomas et al. identified three risk factors were significantly associated with prolonged intubation, including pre-intubation serum bicarbonate $>30 \mathrm{mg} / \mathrm{dl}$ and age $>50$ years [3]. In our cohort, the proportion of patients remaining intubated for longer than 2 weeks was $42.9 \%$ (21/49) with both hypercapnia and age $>50$ years. However, designed prospective study is required verify the statistical significance of various parameters leading to prolonged intubation.

Over $80 \%$ of our patients showed good functional outcome during follow-up. This likely reflects the potentially reversible character of MG and substantial advances in therapeutic and supportive measures [11]. Study has showed that MG is often associated with better functional outcomes at one year than other diseases requiring neurocritical care [25]. However, patients with intermediate and poor outcome had older age of first MC onset, and lower $\mathrm{pH}$ and $\mathrm{PO}_{2}$, as well as higher $\mathrm{PCO}_{2}$ before intubation. Previous study retrospectively included $38 \mathrm{MC}$ patients admitted to the Neuro-medical ICU, and found that 4 patients died in hospital, and the remainder of patients with different age of MC onset (older ( $>50$ years) and younger 
$(<50$ years $)$ patients) did not show differences in longterm outcome [10]. However, one study found that being older than 50 at first MC independently predicted prolonged intubation [3], while another reported that being younger than 40 at MG onset was associated with higher likelihood of remission [13]. The associations between age of first MC onset and outcomes need to be clarified in larger studies with long follow-up. A study showed that preventilation $\mathrm{pH}$ below 7.30 and high $\mathrm{PCO}_{2}$ were associated with poor functional outcome and death [26]. A study comparing MC patients in the ward or in the ICU reported that only those in the ICU had abnormal arterial blood gases, and that patients in the ICU had lower $\mathrm{pH}$ and higher $\mathrm{PCO}_{2}$ [14]. Low $\mathrm{pH}$ and high $\mathrm{PCO}_{2}$ indicate chronic respiratory acidosis, which may be associated with severe disability and death, especially in MG patients who experience MC. Little is known about the outcome of first MC patients suffering from acute severe exacerbations following ICU discharge. In the present study, pre-intubation $\mathrm{PCO}_{2}$ and age of first $\mathrm{MC}$ onset were considered to be factors associated with survival. Therefore, in MC patients with extremely high $\mathrm{PCO}_{2}$ level before intubation may obtain poorer prognosis, especially in patients with older age.

By the end of follow-up, 21 of 113 patients in our cohort had died (18.6\%).This mortality rate is near the high end of the range of 6-30\% reported for MC patients in several studies $[3,9,21,27,28]$. In a Chinese cohort from Hong Kong, 35 MG patients experienced crisis and 2 died (5.7\%) [29], but in a cohort from India, mortality was in 3 out 10 (30\%) during $\mathrm{MC}(30 \%)$ [9]. However, the mortality rate of MC fell from $42 \%$ in the early 1960 s to contemporary rates of 4 to $10 \%$ with the improvement of the advent of IVIg and plasma exchange and ICU management $[3,6]$. The relatively high mortality rate in our study may reflect the fact that we included all consecutive patients who presented in the neurological ICU during the study period. MC patients were managed in the general ward were not included in the present study. Study has shown that compared to MC patients who received general ward management, $\mathrm{MC}$ patients with ICU management had higher MG-ADL scale scores and higher MGFA classification [14]. There could be selection bias, since more seriously ill patients could be selected in the present study. The other fact needed to consider is the ground clinical reality in developing countries, and poor awareness on this part of patients. In addition, drug nonaffordability is the actual reality in China. For example, both plasma exchange and IVIG are not covered by the medical insurance, and the expense on plasma exchange/IVIG is more than the annual income for some Chinese family. In resource-challenged settings like China, vigorous and concerted efforts should be made in $\mathrm{MC}$ prevention, timely identification, emergency intervention, and aggressive treatment.

Our study has several shortcomings. First, study have shown that chronic obstructive pulmonary disease (COPD), diabetes mellitus, atrial fibrillation, hyperlipidemia, myocardial infarction, and malignant tumors, were highly associated with death in the MG population [30]. Another study found that at least one comorbid disease was diagnosed 93\% patients with late-onset MG (after 60 years) [31]. Our results showed that there was no significant difference among patients with different outcomes regarding the comorbidities, probably due to the small sample size and relatively short follow-up time. Second, our population may have been affected by referral bias because our hospital is a tertiary referral center. With regard to hospital-related factors, it is possible that the apparent benefit of neurological care may be far different from other centers. Some management artefact and treatment strategies may lead to changes in outcomes. As a result of the retrospective design of our study, we may have failed to include certain patients who were not entered properly in the hospital computer system. Third, some patients were unable to receive IVIG or plasma exchange due to the family's economic hardship. The usage of immunosuppressants in the treatment of MG has greatly changed the outcome of MG patients [32]. One study indicated that azathioprine therapy independently predicted good clinical outcome of MG patients [29]. Another study showed that combined prednisoloneazathioprine treatment reduced the proportion of recurrent $\mathrm{MCs}$, and the number of mechanical ventilation events and ICU admissions were also reduced [33]. Furthermore, the administration of immunosuppressants was found to be closely associated with a decreased risk for death of MG patients $[29,30]$. However, only 12 patients received immunosuppressants in the present study. These deficiency in treatment may be associated with the poor prognosis in same patients. Finally, we did not analyze data related to other parameters that might have affected clinical outcomes, including maximal expiratory pressure and maximal inspiratory pressure on pulmonary function tests.

\section{Conclusions}

Despite the limitations of our study, our results clearly show that Higher $\mathrm{PCO}_{2}$ prior to mechanical ventilation higher MG-ADL score at $\mathrm{MC}$ onset may be useful indicators of whether a patient has more severe or advanced disease that will likely require extensive respiratory support and ICU management. Higher $\mathrm{PCO}_{2}$, especially in patients who were older at first $\mathrm{MC}$ onset, suggests chronic respiratory acidosis, which may increase risk of severe disability and death. Timely and effective treatment for chronic respiratory acidosis before ICU admission may help prevent exacerbation and improve outcomes. 


\section{Additional file}

Additional file 1: Table S1. Clinical detail of 115 patients with first MC onset requiring ICU treatment. (DOCX $73 \mathrm{~kb}$ )

\section{Abbreviations}

AChR: Acetylcholine receptor; AID: Autoimmune disease; BiPAP: Bilevel positive airway pressure; COPD: Chronic obstructive pulmonary disease; yr., year(s); DM: Diabetic mellitus; h: hour(s); ICU: Intensive care unit; IVIG: Intravenous immunoglobulin therapy; MC: Myasthenic crisis; MG: Myasthenia gravis; MG-ADL: Myasthenia Gravis-Activities of Daily Living scale; MGFA: Myasthenia Gravis Foundation of America scale; mo: month(s); MuSK: Muscle-specific kinase; N/A: Not available; NIF: Negative inspiratory force; PE: Plasma exchange; RNS: Repetitive nerve stimulation; SLE: Systemic lupus erythematosus; VC: Vital capacity; WBC: White blood cell count

\section{Acknowledgments}

The authors thank the patients for their participation in the study.

\section{Authors' contributions}

XPC and FL participated in study design and performed biochemical analyses. XPC drafted the manuscript. XPC performed statistical analysis. XPC conceived the study and assisted in study coordination and manuscript revision. FL and QW collected clinical data and participated in patient care and evaluation. All authors read and approved the final manuscript.

\section{Funding}

The present study was supported by the National Natural Science Foundation of China (grant no. 81301093)

\section{Availability of data and materials}

The raw data summarized in this article are archived at West China Hospital. Although hospital policy prevents their public dissemination out of concern for patient privacy, individual requests for data access may be granted under appropriate circumstances. Interested parties should contact the authors.

\section{Ethics approval and consent to participate}

The study protocol was approved by the Ethics Committee of West China Hospital, Sichuan University. All participants provided written informed consent before being enrolled in the study.

\section{Consent for publication}

As part of their written informed consent to participate in this study, subjects also consented to the publication of their anonymized data for research purposes.

\section{Competing interests}

The authors declare that they have no competing interests.

\section{Author details}

'Department of Neurology, West China Hospital, Sichuan University, 610041, Guoxuexiang \#37, Chengdu, Sichuan, China. ${ }^{2}$ Department of Nursing, West China Hospital of Stomatology, Sichuan University, Chengdu, Sichuan, China. ${ }^{3}$ Neurological Intensive Care Unit, West China Hospital, Sichuan University, Chengdu, Sichuan, China.

Received: 20 November 2018 Accepted: 27 June 2019

Published online: 19 July 2019

\section{References}

1. Deymeer F, Gungor-Tuncer O, Yilmaz V, Parman Y, Serdaroglu P Ozdemir C, Vincent A, Saruhan-Direskeneli G. Clinical comparison of anti-MuSK- vs anti-AChR-positive and seronegative myasthenia gravis. Neurology. 2007;68(8):609-11.

2. Jani-Acsadi A, Lisak RP. Myasthenic crisis: guidelines for prevention and treatment. J Neurol Sci. 2007;261(1-2):127-33.

3. Thomas CE, Mayer SA, Gungor Y, Swarup R, Webster EA, Chang I, Brannagan TH, Fink ME, Rowland LP. Myasthenic crisis: clinical features, mortality, complications, and risk factors for prolonged intubation. Neurology. 1997;48(5):1253-60.
4. Keesey JC. "crisis" in myasthenia gravis: an historical perspective. Muscle Nerve. 2002:26(1):1-3.

5. Chaudhuri A, Behan PO. Myasthenic crisis. QJM. 2009;102(2):97-107.

6. Cohen MS, Younger D. Aspects of the natural history of myasthenia gravis: crisis and death. Ann N Y Acad Sci. 1981;377:670-7.

7. Alshekhlee A, Miles JD, Katirii B, Preston DC, Kaminski HJ. Incidence and mortality rates of myasthenia gravis and myasthenic crisis in US hospitals. Neurology. 2009;72(18):1548-54.

8. Ferguson IT, Murphy RP, Lascelles RG. Ventilatory failure in myasthenia gravis. J Neurol Neurosurg Psychiatry. 1982;45(3):217-22.

9. Sharma S, Lal V, Prabhakar S, Agarwal R. Clinical profile and outcome of myasthenic crisis in a tertiary care hospital: a prospective study. Ann Indian Acad Neurol. 2013:16(2):203-7.

10. Spillane J, Hirsch NP, Kullmann DM, Taylor C, Howard RS. Myasthenia gravis--treatment of acute severe exacerbations in the intensive care unit results in a favourable long-term prognosis. Eur J Neurol. 2014; 21(1):171-3.

11. Bershad EM, Feen ES, Suarez J. Myasthenia gravis crisis. South Med J. 2008;101(1):63-9.

12. Wang W, Chen YP, Wang ZK, Wei DN, Yin L. A cohort study on myasthenia gravis patients in China. Neurol Sci. 2013;34(10):1759-64.

13. Mantegazza R, Baggi F, Antozzi C, Confalonieri P, Morandi L, Bernasconi $P$, Andreetta F, Simoncini O, Campanella A, Beghi $E$, et al. Myasthenia gravis (MG): epidemiological data and prognostic factors. Ann N Y Acad Sci. 2003;998:413-23.

14. Sakaguchi H, Yamashita S, Hirano T, Nakajima M, Kimura E, Maeda Y, Uchino M. Myasthenic crisis patients who require intensive care unit management. Muscle Nerve. 2012;46(3):440-2.

15. Bae JS, Go SM, Kim BJ. Clinical predictors of steroid-induced exacerbation in myasthenia gravis. J Clin Neurosci. 2006;13(10):1006-10.

16. Tomiyama M, Arai A, Kimura T, Suzuki C, Watanabe M, Kawarabayashi T, Shoji M. Exacerbation of chronic pancreatitis induced by anticholinesterase medications in myasthenia gravis. Eur J Neurol. 2008;15(5):e40-1.

17. Godoy DA, Mello LJ, Masotti L, Di Napoli M. The myasthenic patient in crisis: an update of the management in Neurointensive care unit. Arq Neuropsiquiatr. 2013;71(9A):627-39.

18. Lacomis D. Myasthenic crisis. Neurocrit Care. 2005;3(3):189-94.

19. Plauche WC. Myasthenia gravis in mothers and their newborns. Clin Obstet Gynecol. 1991;34(1):82-99.

20. Gracey DR, Divertie MB, Howard FM Jr. Mechanical ventilation for respiratory failure in myasthenia gravis. Two-year experience with 22 patients. Mayo Clin Proc. 1983;58(9):597-602

21. Panda S, Goyal V, Behari M, Singh S, Srivastava T. Myasthenic crisis: a retrospective study. Neurol India. 2004;52(4):453-6.

22. Perlo VP, Poskanzer DC, Schwab RS, Viets HR, Osserman KE, Genkins G. Myasthenia gravis: evaluation of treatment in 1,355 patients. Neurology. 1966;16(5):431-9.

23. Seneviratne J, Mandrekar J, Wijdicks EF, Rabinstein AA. Noninvasive ventilation in myasthenic crisis. Arch Neurol. 2008;65(1):54-8.

24. Rabinstein A, Wijdicks EF. BiPAP in acute respiratory failure due to myasthenic crisis may prevent intubation. Neurology. 2002;59(10):1647-9.

25. Kiphuth IC, Schellinger PD, Kohrmann M, Bardutzky J, Lucking H, Kloska S, Schwab S, Huttner HB. Predictors for good functional outcome after neurocritical care. Crit Care. 2010:14(4):R136.

26. Cabrera Serrano M, Rabinstein AA. Usefulness of pulmonary function tests and blood gases in acute neuromuscular respiratory failure. Eur J Neurol. 2012;19(3):452-6.

27. Werneck LC, Scola RH, Germiniani FM, Comerlato EA, Cunha FM. Myasthenic crisis: report of 24 cases. Arq Neuropsiquiatr. 2002;60(3-A):519-26.

28. Murthy JM, Meena AK, Chowdary GV, Naryanan JT. Myasthenic crisis: clinical features, complications and mortality. Neurol India. 2005;53(1): 37-40 discussion 40.

29. Lee CY, Lam CL, Pang SY, Lau KK, Teo KC, Chang RS, Chan KH. Clinical outcome of generalized myasthenia gravis in Hong Kong Chinese. $J$ Neuroimmunol. 2015:289:177-81.

30. Liu C, Wang Q, Qiu Z, Lin J, Chen B, Li Y, Gui M, Zhang M, Yang M, Wang W, et al. Analysis of mortality and related factors in 2195 adult myasthenia gravis patients in a 10-year follow-up study. Neurol India. 2017:65(3):518-24.

31. Fraisse $T$, Labauge $P$, Camu W, Arlaud $P$, de Wazieres B. Myasthenia gravis in the elderly: diagnosis, comorbidity and course: 45 cases. Presse Med. 2007; 36(1 Pt 1):9-14 
32. Hoffmann S, Kohler S, Ziegler A, Meisel A. Glucocorticoids in myasthenia gravis - if, when, how, and how much? Acta Neurol Scand. 2014;130(4):211-21.

33. Rozsa C, Mikor A, Kasa K, Illes Z, Komoly S. Long-term effects of combined immunosuppressive treatment on myasthenic crisis. Eur Neurol. 2009;16(7):796-800.

\section{Publisher's Note}

Springer Nature remains neutral with regard to jurisdictional claims in published maps and institutional affiliations.

Ready to submit your research? Choose BMC and benefit from:

- fast, convenient online submission

- thorough peer review by experienced researchers in your field

- rapid publication on acceptance

- support for research data, including large and complex data types

- gold Open Access which fosters wider collaboration and increased citations

- maximum visibility for your research: over $100 \mathrm{M}$ website views per year

At $B M C$, research is always in progress.

Learn more biomedcentral.com/submissions 